\title{
Chronic Xiao-chai-hu Decoction Therapy Slows the Progression of Acute-on-chronic Liver Failure via Prokinetic Effects on Gastrointestinal Motility
}

\section{Feng Ye}

Xi'an Jiaotong University Medical College First Affiliated Hospital

Jian Zhang

Shaanxi Provincial People's Hospital

\section{Yi-long Wang}

Xi'an Jiaotong University Medical College First Affiliated Hospital

Shu-mei Lin

Xi'an Jiaotong University Medical College First Affiliated Hospital

\section{Xi Zhang}

Xi'an Jiaotong University Medical College First Affiliated Hospital

\section{Xiao-jing Liu}

Xi'an Jiaotong University Medical College First Affiliated Hospital

Jian-zhou Li

Xi'an Jiaotong University Medical College First Affiliated Hospital

yi liu ( $\square$ liuyi.jiaotong@163.com )

Xian Jiaotong University: Xi'an Jiaotong University

\section{Research}

Keywords: acute-on-chronic liver failure, Xiao Chai Hu decoction, systemic inflammation, liver damage, apoptosis, gastrointestinal motility

Posted Date: October 12th, 2021

DOl: https://doi.org/10.21203/rs.3.rs-936610/v1

License: (c) (i) This work is licensed under a Creative Commons Attribution 4.0 International License. Read Full License 


\section{Abstract}

\section{Background}

Acute-on-chronic liver failure (ACLF) has a high risk of mortality in liver diseases without effective treatment. Xiao Chai Hu decoction (XCHD) is a traditional herbal formula, widely administered for liver disease, including anti-hepatic fibrosis and anti-inflammatory.

\section{Methods}

To investigate whether XCHD prevents the progression of ACLF and the underlying mechanisms. Methods: Sprague-Dawley rats with compound factors were randomly divided into model control group with 30 rats and four treatment groups with 20 rats in each group: Polyene phosphatidylcholine (100 $\mathrm{mg} / \mathrm{kg}$; PP) group; high dose (44.5 g/kg; HXCHD), middle dose (26.5 g/kg; MXCHD), and low dose (8.5 $\mathrm{g} / \mathrm{kg} ; \mathrm{LXCHD}$ ). Firstly, liver disease progression after XCHD treatment was measured by detecting: 1) the serum levels of aspartate aminotransferase (AST), alanine aminotransferase (ALT), total bilirubin (TB), and albumin, prothrombin time (PT);2) the survival rate and rat-adapted model for end-stage liver disease (MELD) score; 3 ) the hepatic hyperplastic collagen fibers with Masson's trichrome staining; 4) the serum level of TNF-a and endotoxin induced hepatocyte apoptosis expression; 5) the ileum slow waves, gastric emptying, and small intestinal transit after XCHD treatment.

Results

The XCHD groups showed improved serum biochemical hepatic parameters, histological liver changes, and survival rate. In addition, the XCHD treatment decreased the serum level of TNF-a and endotoxin and reduced hepatocyte apoptosis. XCHD decreased the normal percentage ileum slow waves, prolonged gastric emptying, and increased the small intestinal transit of ACLF.

\section{Conclusions}

$\mathrm{XCHD}$ prevented the progression of ACLF partially via the prokinetic effects on gastrointestinal motility to reduce TNF-a, endotoxin, and hepatocyte apoptosis.

\section{Background}

Acute-on-chronic liver failure (ACLF) is a syndrome in patients with chronic liver disease with or without previously diagnosed cirrhosis. It is characterized by acute hepatic decompensation resulting in liver failure and one or more extrahepatic organ failures. Despite the application of extracorporeal liver support systems, such as plasma exchange, molecular adsorbent recirculating system, and liver transplantation in recent years (Finkenstedt et al., 2013), the mortality rate of ACLF is still 30-50\% (Solé et al., 2018). Notably, $25 \%$ of the ACLF patients are suitable for a liver transplant (Finkenstedt et al., 2013). Moreover, a randomized study showed a significant increase in the survival of extracorporeal liver support systems (Bañares et al., 2013; Kribben et al., 2012). Other potential therapies for improving liver function, such as 
cell transplantation (Yuan et al.; Huebert et al., 2014) and colony-stimulating factor (CSF) (Khanam et al., 2014), are rather challenging in both developing and developed countries due to heavy medical expenses. Hitherto, the curative effect of ACLF is limited (Solé et al., 2018), thereby necessitating effective therapeutic methods.

Chinese herbal medicine has been adopted in the treatment of liver diseases for a long time in China (Qi et al., 2013). Xiao Chai Hu decoction (XCHD) is widely recognized as a medicine in Asia for the treatment of liver diseases. It is a well-known prescription in traditional Chinese medicine (TCM), and is composed of seven herbs: Radix bupleuri, Scutellaria baicalensis, Panax ginseng, Rhizoma Pinelliae preparata, honey-fried Glycyrrhizae Radix, Zingiber officinale, and Ziziphus jujube (Xiong et al., 2011). It was believed that XCHD alleviates the symptoms of fever, abdominal distension, poor appetite, nausea, vomiting, and jaundice. Previous studies confirmed that XCHD inhibits the progression of hepatitis to liver fibrosis (Dou et al., 2005) and the replication of hepatitis B and C virus (Li et al., 2017). Moreover, it prevents liver damage via immune regulation (Zheng et al., 2014; Liu et al., 2013). Bupleurum and Scutellaria baicalensis are the core paired components of this prescription. Previous studies have shown that Scutellaria root is effective an antiendotoxic (Cheng et al., 2007), anti-bacterial (Miyasaki et al., 2013), anti-hepatic fibrosis (Miyasaki et al., 2013; Qiao et al., 2011), anti-inflammatory (Pan et al., 2012; Shen et al., 2003; Kubo et al., 1984; Yang et al., 2009; Yun et al., 2010), antiviral (Dou et al., 2012) agent. Cirrhotic endotoxemia is a major factor causing disease progression and multiple organ failure. Moreover, XCHD alleviates chronic liver damage that might decrease the mortality risk of patients. Thus, we hypothesized that XCHD prevents ACLF-induced death via prokinetic effects on the gastrointestinal motility against tumor necrosis factor-alpha (TNF-a) and endotoxin-induced hepatocyte apoptosis.

\section{Methods}

\subsection{Experimental animals}

A total of 120 healthy and clean male Sprague-Dawley rats $(90-120 \mathrm{~g})$ were purchased from the Laboratory Animal Center of Xi'an Jiao Tong University (Xi'an, Shaanxi, China), acclimated to the research laboratory for five days before experiments, and maintained in a light-controlled room (12 h light/dark cycle) at an ambient temperature of $25^{\circ} \mathrm{C}$ with free access to water and standard chow. The experiments were conducted according to the Guide for the Care and Use of Laboratory Animals (Institute of Laboratory Animal Resources, 1996, Nat. Acad. Press) and approved by the Xi'an Jiao Tong University (XJTU20121210009) Institutional Animal Care and Use Committee (IACUC).

\subsection{Experimental materials}

Seven crude herbs and polyene phosphatidylcholine (Sanofi, China Pharmaceutical Co., Ltd) were purchased from the pharmacy of the First Affiliated Hospital of Xi'an Jiao Tong University.

Lipopolysaccharide (LPS from E. coli 055:B5) was purchased from Sigma-Aldrich Chimie Company (Fallavier, France). Apoptosis detection kit was obtained from Biosynthesis Biotechnology Co., Ltd (Beijing, China). Carbon tetrachloride analytical reagent (AR) was purchased from Chemical Reagent 
Branch of Tianjin Zonghengxing Industrial and Trading Co., Ltd. Olive oil AR was purchased from Sinopharm Chemical Reagent Co., Ltd (Shanghai, China). Rat endotoxin quantitative detection kit was procured from Beijing Jinshanchuan Technology Development Co., Ltd. Isoflurane was purchased from Forane, Abbott Laboratories, Abbott Park, IL, USA. The cardiac pacing wires were obtained from Medtronic, Minneapolis, MN, USA. The aspartate aminotransferase (AST), alanine aminotransferase (ALT), total bilirubin (TB), albumin (ALB), and prothrombin time (PT) commercial kits were purchased from Sigma-Aldrich, USA.

\subsection{Experimental methods}

\subsubsection{Reagent preparation}

1) CCL4 oil solution preparation: A volume of $40 \mathrm{~mL}$ CCL4 was solubilized in $60 \mathrm{~mL}$ olive oil; and $40 \%$ CCL4 oil solution was prepared and stored at room temperature.

2) XCHD (Su et al., 2014): It consisted of seven crude herbs (herbs implementing standards:

Pharmacopoeia of the People's Republic of China 2015 first edition). These herbs were mixed and decocted three times $(100 \mathrm{~g} / 1000 \mathrm{~mL}$ the first time; $100 \mathrm{~g} / 400 \mathrm{~mL}$ the second time; $100 \mathrm{~g} / 400 \mathrm{~mL}$ the third time) with water for $30 \mathrm{~min}$, as described previously. The decoction was filtered through the gauze; the filtrate was concentrated as $4.45 \mathrm{~g} / \mathrm{mL}$.

Table 1. Herbs of XCHD

\begin{tabular}{|c|c|c|c|}
\hline $\begin{array}{l}\text { Chinese } \\
\text { name }\end{array}$ & Botanical name & Lot no. & $\begin{array}{l}\text { Dosage } \\
\text { (g/day) }\end{array}$ \\
\hline Chaihu & $\begin{array}{l}\text { Radix Bupleurum scorzonerifolium } \\
\text { Willd. }\end{array}$ & $\begin{array}{l}\text { 20160408; Shannxi, } \\
\text { China }\end{array}$ & 12 \\
\hline Huangqin & Scutellaria baicalensis Georgi & $\begin{array}{l}\text { C3022004001; Shanxi, } \\
\text { China }\end{array}$ & 9 \\
\hline Banxia & Pinellia ternata (Thunb.) Makino & 343200401; Gansu, China & 9 \\
\hline Renshen & Panax ginseng C. A. Mey & 343200401; Jilin, China & 9 \\
\hline Zhigancao & $\begin{array}{l}\text { honey-fried Radix Glycyrrhiza uralensis } \\
\text { Fisch }\end{array}$ & 20160301; Gansu, China & 6 \\
\hline Shengjiang & Zingiber officinale Roscoe & $\begin{array}{l}\text { C0512005001; Yunnan, } \\
\text { China }\end{array}$ & 6 \\
\hline Dazao & Ziziphus jujuba Mill & $\begin{array}{l}\text { 20160112; Xinjiang, } \\
\text { China }\end{array}$ & 9 \\
\hline
\end{tabular}

\subsubsection{Protocol for surgery}

After an overnight fast, under anesthesia with the inhalation of $1.5-2.0 \%$ isoflurane, the hair was shaved, the skin on top of the corresponding position was cut open, and then one pair of cardiac pacing wires 
was implanted in the serosal surface of the ileum about $4 \mathrm{~cm}$ proximal to the ileocecum for ileum slowwave recording. The distance between the two electrodes in the pair was $0.3 \mathrm{~cm}$. After the isolated lead wires were tunneled and externalized on the rat's neck, buprenorphine $(0.05 \mathrm{mg} / \mathrm{kg})$ and cefazolin (30 $\mathrm{mg} / \mathrm{kg}$ ) was administered for 2 days to alleviate postoperative pain and prevent infection, respectively. The rats were housed individually to avoid the wires and tubes from being chewed off by other rats. The experiments were initiated after the rats were completely recovered from the surgery, usually 7 days after the operation.

\subsubsection{Protocol for the making of CCL4-induced ACLF model (Figure 1)}

After 7 days of conventional adaptive feeding, 110 rats were used to establish the model. Then, 10 rats were used as the normal control group. During the initial 4-week treatment, $40 \%$ CCL4 olive oil solution was injected subcutaneously at $1.5 \mathrm{~mL} / \mathrm{kg}$ body weight every three days. Then, in the following two weeks, the $40 \%$ CCL4 olive oil solution was injected subcutaneously at $2 \mathrm{~mL} / \mathrm{kg}$ body weight every three days. After the establishment of the cirrhotic rat model, lipopolysaccharide (LPS)- and D-galactosamine (D-Gal)-induced ACLF models were constructed. On day 45, the rats were injected LPS at a dose of $10 \mathrm{mg} / \mathrm{kg}$ and D-Gal at a dose of $700 \mathrm{mg} / \mathrm{kg}$ intraperitoneally, which induce acute liver failure based on chronic liver injury.

The normal control rats were administered physiological saline at the time points same as in the model rats. The animal behavior was closely monitored during the modeling.

\subsubsection{Method of administration}

During modeling, 110 rats were randomly divided into model control group with 30 rats and four treatment groups with 20 rats in each group: polyene phosphatidylcholine $(100 \mathrm{mg} / \mathrm{kg} ; \mathrm{PP})$ group, highdose (44.5 g/kg; HXCHD), middle-dose (26.5 g/kg; MXCHD), and low dose ( $8.5 \mathrm{~g} / \mathrm{kg} ; \mathrm{LXCHD)} \mathrm{Xiao} \mathrm{Chai}$ Hu decoction. In the model and control groups, rats were given an equivalent volume of water by gavage daily.

\subsubsection{Specimen collection and preparation}

On day 44, five rats were selected randomly in the ACLF model group, and their liver histopathology (included) was detected to determine the cirrhosis. After an acute attack, $0.6 \mathrm{~mL}$ blood samples withdrawn at $2 \mathrm{~h}, 10 \mathrm{~h}$, and $18 \mathrm{~h}$ were collected from the inner canthus in all rats to detect the serum biochemical levels. After 47 days of treatment, the colon slow-wave was measured. Then, all the surviving rats were sacrificed for specimen collection. One part of the liver tissue was cut and embedded in paraffin for hematoxylin and eosin (H\&E) and Masson's trichrome staining.

\subsubsection{Analysis of liver disease progression}

1) Serum levels of liver functionality measurements 
The serum was obtained by centrifugation of blood samples at $1500 \times g$ for $10 \mathrm{~min}$ at $4{ }^{\circ} \mathrm{C}$ and stored at room temperature for $1 \mathrm{~h}$ and then at $20^{\circ} \mathrm{C}$ until further analysis. The activity of AST, ALT, TB, ALB, and PT was measured using commercially available kits (Sigma-Aldrich), according to the manufacturer's instructions.

2) Survival rate and rat-adapted model of end-stage liver disease (MELD) score (Said et al., 2004)

Based on the literature, the rats' MELD was adapted as follows: Rat-adapted MELD score $=0.957 \times$ Loge (creatinine $\mathrm{mg} / \mathrm{dL}$ ) $+0.378 \times$ Loge (bilirubin $\mathrm{mg} / \mathrm{dL}$ ) $+1.120 \times$ Loge $($ INR $)+0.643$. MELD is a scale system to score the severity of liver disease and predict death. A high MELD score indicates a high probability of death.

3) H\&E staining, Masson's trichrome staining, and image analysis

The H\&E staining and Masson's trichrome staining revealed hepatic lobule structures and hepatic cords. In Masson staining, blue color indicated hyperplastic collagen fibers. Five low-power $(\times 40)$ fields were randomly assessed for each slice. The ratio of the area of hepatic hyperplastic collagen fibers (AHHCF\%) was calculated by Image Pro-Plus 6.

\subsubsection{Mechanisms of XCHD involving endotoxin-induced hepatocyte apoptosis}

1) Detection of serum endotoxin and TNF-a levels with two-step double-antibody sandwich enzyme-linked immunosorbent assay (ELISA).

According to the manufacturer's description $2.5-80 \mathrm{pg} / \mathrm{mL}$ was used in ELISA to detect and quantitatively analyzed the serum endotoxin and TNF-a levels of rats in all the groups. A volume of 100 $\mu \mathrm{L}$ samples was dispensed in each well in 96-well plates after pre-coating with rat TNF-a/endotoxin antibody for $30 \mathrm{~min}$ at $37^{\circ} \mathrm{C}$. Then, a protein-specific biotinylated antibody was incubated for $2 \mathrm{~h}$ at 37 ${ }^{\circ} \mathrm{C}$. Between each reaction, the unbound proteins were washed away from the wells. Then, substrate solutions $A$ and $B$ were added to each well, and the reaction incubated for $20 \mathrm{~min}$ at $37^{\circ} \mathrm{C}$. The absorbance was measured at $450 \mathrm{~nm}$ in a microplate reader, and the concentration data expressed as a mean value.

2) Detection of hepatocyte apoptosis by terminal deoxynucleotide transferase dUTP nick end labeling (TUNEL)

According to the manufacturer's instructions, TUNEL method was used to detect cellular apoptosis on the liver tissue. The sections were fixed in ethanol-acetic acid (2:1), incubated with proteinase $\mathrm{K}(100 \mu \mathrm{g} / \mathrm{mL})$, rinsed in PBS, incubated in $3 \% \mathrm{H}_{2} \mathrm{O}_{2}$, and washed with phosphate-buffered saline (PBS) for $10 \mathrm{~min}$, followed by permeabilization ( $0.1 \%$ Triton $X-100,0.1 \%$ sodium citrate) for 5 min. Subsequently, the sections were washed and incubated in TUNEL reaction mixture. Converter-POD with $0.02 \% 3,3$ 'diaminobenzidine (DAB) was used for visualization, and Mayer's hematoxylin was used for counterstaining. TUNEL-positive cells were dyed as the yellow nucleus. The liver cells were counted under high- 
power $(\times 400)$ field. Five high-power fields were examined in each case, and the number of TUNEL-positive cells was counted. Finally, 500 cells were counted in each field, and the apoptotic index (Al\%) of each group was calculated.

\subsubsection{Mechanisms of XCHD involving gastrointestinal motility}

After overnight fasting, each rat was fed $2 \mathrm{~g}$ of solid dry food at the end of the experiment. A volume of $1.5 \mathrm{~mL}$ phenol red $(0.5 \mathrm{mg} / \mathrm{mL})$ was mixed with $1.5 \%$ methylcellulose was delivered by gavage after 10 min. Then, the rat was euthanized, the content of the stomach and the small intestine was collected for measuring the gastric emptying (GE) (Lin et al., 2018) and small intestinal transit (SIT) (Ohno et al., 2006), using a previously established method.

The percentage of GE was described as the ratio between the amount of undigested food in the stomach and $2 \mathrm{~g}$ of solid dry food. The small intestine was cut into 10 equal segments. The SIT was assessed using the geometric center based on the amount of phenol red in each of the segments (Scarpignato et al., 1980).

\subsection{Statistical methods}

SPSS16.0 statistical analysis software package (IBM, Armonk, NY, USA) was utilized. Continuous data were expressed as mean \pm standard. One-way analysis of variance (ANOVA) was performed, and q test (Student-Newman-Keuls test, S-N-K) was used for pairwise comparisons. Kaplan-Meier method was used to calculate the survival rate. Two-sided P-values $<0.05$ were considered statistically significant.

\section{Results}

\subsection{Verification of ACLF rat model}

After the abdominal cavity was opened, it was overflowed with turbid yellow liquid. Varying degrees of adhesion was observed between the abdominal tissues. The surface of the liver was rough and uneven, and dense nodules were detected in the cirrhosis and ACLF model (Figure 2B, C). Also, white fur or pus was on the surface of the liver in the ACLF model than the cirrhosis model.

H\&E staining and Masson's trichrome staining detected clear hepatic lobule structures in the normal rats (Figure 3A1, A2). In the model group, the hepatic lobule structures were damaged, and liver cell cords were arranged disorderly with obvious swelling and fatty degeneration (Figure 3B1, B2, C1, C2). A large number of blue hyperplastic thick collagen fibers was observed in the portal area and lobules in Masson staining. The hepatic cell cords of the liver lobule were disordered, and a large amount of unequal-sized pseudo lobules were formed. Also, a large number of necrotic liver cells were detected, and inflammatory cell infiltration was observed (Figure 3C1, C2).

After acute attachment for $2 \mathrm{~h}$, the serum level of ALT, AST, and TNF-a increased sharply and decreased in $10 \mathrm{~h}$. However, the level of total bilirubin (TBIL) increased progressively from 2-18 h (Figure 4A), 
indicating liver failure. Together, these findings demonstrate an experimental model of ACLF.

\subsection{Comparison of the liver disease progression}

1) Comparison of the serum levels of liver functionality measurements

The activity of AST, ALT, TB, ALB, and PT was measured using commercially available kits (SigmaAldrich), according to the manufacturer's instructions.

Compared to the normal control group, the experimental groups showed a significantly increase in the levels of ALT, AST, and TBil $(P<0.05, F=4.15)$. The serum ALT, AST, and TBil levels of the model group (839.6 $\pm 55.4 \mathrm{IU} / \mathrm{L} ; 852.0 \pm 66.2 \mathrm{IU} / \mathrm{L} ; 153.1 \pm 5.2 \mathrm{mmol} / \mathrm{L})$ were higher those of the all the XCHD treatment groups, (P-values: ALT < 0.01, AST < 0.01, DBil < 0.01, TBil < 0.01). The serum ALT, AST, and TB levels of the HXCHD treatment group were the lowest among the three treatment groups (Figure 4B, 4C). These data suggested that XCHD treatment improves hepatobiliary function. Importantly, the serum ALB levels of the rat model demonstrated a significant decrease, whereas the animals treated with HXCHD and MXCHD showed a similar increase compared to those of the PP group (Figure 4D). This demonstrated that HXCHD promotes liver functionality as a protein producer. The rats treated with XCHD showed decreased PT (Figure 4E). Conversely, no differences were detected in the PT among the PP, HXCHD, and MXCHD groups.

2) Comparison of the survival rate and rat-adapted MELD score

Two stages were detected in the ACLF model. The first lasted 4 weeks to develop progressive chronic liver failure; six rats died in the model group, five rats died in LXCHD, four rats died in MXCHD, and none of the rats died in the HXCHD groups. In addition to chronic liver failure, acute liver failure resulted in rat mortality. After $48 \mathrm{~h}$ of acute attack, the survival rate of the various groups was as follows: $100 \%$ in the normal control group, in the model and LXCHD groups $\left(X^{2}=0.078, P<0.05 v s\right.$. model), $77 \%$ in the $\operatorname{MXCHD}$ group $\left(X^{2}=1.99, P<0.05\right.$ vs. model $)$, and $60 \%$ in the HXCHD group $\left(X^{2}=11.37, P<0.05 v s\right.$. model; $\chi^{2}=5.976, P<0.05$ vs. MXCHD). We also observed the time of death. In the model group, the death mainly occurred during the chronic liver failure stage to a relatively high mortality rate of $30 \%$, while early survival was $100 \%$ in the HXCHD-treated rats (Figure 5A). After administration of D-gal/LPS, the $\mathrm{HXCHD}$ group succumbed to mortality at $20 \mathrm{~h}$ compared to that in the model group at $12 \mathrm{~h}$, suggesting that treatment with XCHD effectively slowed the evolution of the disease.

In addition, the XCHD treated groups showed a significantly lower MELD score than the model group (Figure 5B), indicating healthier livers after XCHD treatment. Taken together, this phenomenon suggested that XCHD treatment slowed the evolution of the disease. Compared to the model rats (Figure 5C), the INRs were decreased in the rats treated with XCHD compared to the model group similar to PP. These data indicated an improvement on liver failure due to XCHD treatment.

3) Comparison of the ratio of the area of hepatocyte apoptosis in each group 
Hepatocyte apoptosis in normal rats was detected occasionally (Figure 6A). The hepatocyte apoptosis of ACLF rats increased markedly. The positive nuclei with brown or black color were scattered diffusely in the field and were pyknotic-like, or chromatin aggregated to the surrounding, or the nuclei were fragmented (Figure 6B).

The hepatocyte apoptosis rate of the experimental groups was significantly higher than that of the normal control group $(P<0.01, F=29.03)$. The hepatocyte apoptosis rate of the model group $(8.8 \pm 0.8)$ was significantly higher than that of the HXCHD $(2.92 \pm 0.26)$ group, the MXCHD $(3.9 \pm 0.5)$ group, and the LXCHD $(5.8 \pm 0.3)$ group $(P<0.05$; Figure 6C, 6D, 6E, 6F).

4) Comparison of the ratios of the area of hepatic hyperplastic collagen fibers in each group

The model rats showed $29.8 \%$ area of fibrosis in the pathological livers, while improved hepatic histology was observed in XCHD rats. The hepatic fibrogenesis fibrosis was significantly decreased in the XCHDtreated rats as follows (Figure 7A): in the HXCHD (10.88 $\pm 0.88 ; \mathrm{P}<0.05$ vs. model), MXCHD (15.32 \pm $0.55 ; \mathrm{P}<0.05$ vs. model), and LXCHD (19.18 $\pm 1.12 ; \mathrm{P}<0.05$ vs. model) groups. Moreover, the percentage of fibrosis was significantly lower in the HXCHD rats than in the rats treated with $\mathrm{PP}(12.14 \pm 0.90 ; \mathrm{P}<$ 0.05 vs. model).

The pooled biochemical and histopathology data indicated that the administration of HXCHD slowed the progression of the disease. Subsequent studies investigated whether the low survival rate of XCHD was associated with TNF-a and endotoxin-induced hepatocyte apoptosis.

5) Comparison of the serum levels of TNF-a and endotoxin in each group

Compared to the normal control group, the TNF- $a$ and endotoxin levels of all the experimental groups increased significantly $\left(P<0.05, F_{T N F-a}=4.37 ; F_{\text {endotoxin }}=3.31\right.$; Figure 7B, $\left.C\right)$. The serum TNF-a and endotoxin levels of the model group were significantly higher than those of all the treatment groups. The serum endotoxin level of the HXCHD treatment group was significantly lower than that of the PP treatment group, $\left(P<0.05 ; q_{T N F-a}=3.98 ; q_{\text {endotoxin }}=2.79\right)$.

6) Prokinetic effects of XCHD on motility

In ACLF rats, the GE $(36.5 \pm 5.8)$ and SIT $(11.4 \pm 2.3)$ were delayed compared to the control group (78.1 \pm 8.6; 7.6 $\pm 1.0, \mathrm{P}<0.05$ ). XCHD significantly accelerated GE (HXCHD: $66.7 \pm 9.3$; MXCHD: $51.1 \pm 7.9$; LXCHD: $42.3 \pm 6.7$; P 0.05; Figure 8A) and SIT (HXCHD: $8.5 \pm 0.9$; MXCHD: $9.6 \pm 0.7$; LXCHD: $10.8 \pm 0.8$; $\mathrm{P}<0.05$; Figure 8B). It indicated that $\mathrm{XCHD}$ prevented $\mathrm{ACLF}$-induced impairment of gastrointestinal motility.

Compared to the normal group, the percentage of normal ISW (intestinal slow wave) was substantially reduced in the ACLF model. The HXCHD treatment prevented the ACLF-associated decline in the percentage of normal ISW (Figure 9). 


\section{Discussion}

ACLF is one of the leading causes of deaths in patients with chronic liver diseases. In recent years, some progress has been made to decrease the mortality rate of ACLF, such as using the combination of antivirus, artificial liver, and liver transplantation. However, medical expenses, expensive medical equipment, and limited donor organ hinder development, especially in the developing country.

Thus, new strategies are urgently required for high mortality. In the present study, XCHD was beneficial in treating liver fibrosis, which improved the serum parameters of liver disease. In addition, the preventive therapy with XCHD satisfactorily improved the cirrhosis and ACLF survival in rats. Furthermore, XCHD prevented ACLF via a decline in TNF-a and endotoxin-induced hepatocyte apoptosis through the prokinetic effects on gastric motility.

Based on the "two-hits" theory (Amacher et al., 2011; Hernández-Muñoz et al., 1997; Weber et al., 2003), low dose and prolonged $\mathrm{CCl} 4$ administration extensively induces liver fibrosis (Furuya et al., 2016) and with D-gal/LPS injection induces ACLF. In this model, we demonstrated that liver fibrosis/cirrhosis is induced by $\mathrm{CCl} 4$, which is confirmed by liver histology results. $\mathrm{CCl} 4$ leads to the necrosis of hepatocytes, induces inflammation, and further promotes the progression of hepatic fibrogenesis in rats. Subsequent D-gal/LPS treatment caused acute massive hepatic necrosis, and a large white fur or pus was observed on the surface of the liver in ACLF rats.

ACLF progresses rapidly, and its mortality rate in patients is $30-40 \%$ (Arroyo et al., 2015). Reportedly, during the fibrosis-induction period, the CCL4-induced model showed high mortality of $30 \%$, and about $90 \%$ of the rats died from acute liver failure after administration of D-gal/LPS within $13 \mathrm{~h}$ (Liu et al., 2007). In this study, a high mortality rate was observed within a short period following D-gal/LPS treatment, starting after $12 \mathrm{~h}$ and reaching $100 \%$ at $48 \mathrm{~h}$. The subsequent findings on the significantly increased levels of ALT and AST that decreased afterwards, and the markedly increased TB in the ACLF model indicated liver parenchymal damage. Taken together, these observations suggested that ACLF rat model reflects the typical clinical manifestations of ACLF.

ACLF with cirrhosis shows a higher mortality rate in acute decompensation than without cirrhosis (RuizMargáin et al., 2018; Arroyo et al., 2016). Hitherto, the occurrence of liver failure and inflammation damage should be prevented in liver fibrosis, which would be an alternative option for treating ACLF. The current data showed that HXCHD promotes liver functionality as a protein producer. Consistent with previous studies (Li et al., 2017), XCHD exerted a protective effect on inhibiting the formation of hepatic fibrosis, which further showed a significant dose-effect correlation of XCHD on inhibiting the formation of hepatic fibrogenesis fibrosis.

Although the mechanisms of ACLF are not precisely delineated, these are associated with infectioninduced systemic inflammation, acute liver damage, and intestinal location of bacteria or bacterial products (Liu et al., 2007; Arroyo et al., 2016). The poor outcome of ACLF is closely associated with exacerbated systemic inflammatory responses (Clària et al., 2016). A previous study showed that CCl4 
attacks hepatocytes and causes necrosis of parenchymal cells, which in turn promotes inflammatory responses in the liver. The increased levels of ALT and AST serve as markers in detecting acute and chronic liver injury (Gressner et al., 2007); moreover, the serum activities are associated with liver parenchymal damage. Also, serum total bile acid could serve as an additional marker for risk stratification in cirrhotic patients with ACLF (Wu et al., 2018; Horvatits et al., 2017). In the current study, all doses of XCHD decreased the serum levels of ALT, AST, and TB.

Accumulating evidence has shown that hepatocyte apoptosis is the predominant mode of cell death in ACLF (Adebayo et al., 2015; Cai et al., 2016; Bechmann et al., 2008; Rastogi et al., 2011) and plays a role in the pathogenesis of ACLF. Moreover, apoptosis vs. necrosis rate would serve as a predictor in acute liver failure compared with acute-on-chronic liver failure (Bechmann LP et al., 2008). Some toxic molecules induced hepatocyte apoptosis and worsened the liver disease through caspase-8 and caspase3-dependent pathways, as assessed in the liver failure plasma of the patients. A therapeutic strategy was proposed to remove these pro-apoptotic factors. Hitherto, no study has described a molecular adsorbent recirculation system dialysis to reduce these toxic molecules in the presence of liver failure plasma (Saich et al., 2007). In the current study, with different doses of XCHD treatment, the hepatocyte apoptosis rate was significantly reduced, while the survival rate was increased after XCHD treatment within $48 \mathrm{~h}$. However, it was challenging to determine whether XCHD removed the pro-apoptotic factors from the plasma directly and whether hepatocyte apoptosis is closely related to the survival from ACLF.

TNF-a is a major cytokine produced by inflammatory cells (Baggiolini, 2001). Among the clinical TCMs, $\mathrm{XCHD}$ has been widely used in treating chronic hepatitis, cirrhosis, and acute and chronic cholecystitis ( $\mathrm{Li}$, 2020). Accumulating experimental and clinical evidence suggested that XCHD affects liver diseases via an anti-inflammatory mechanism, suppressing the progression of liver damage and via immune regulation (Qi et al., 2013; Dou et al., 2005; Zheng et al., 2014; Liu et al., 2013). In correspondence with the previous findings, we demonstrated that all doses of XCHD significantly decreased the TNF-a levels, which also verified the anti-inflammatory effect of XCHD. These findings indicated that the XCHD exerted an anti-inflammatory effect, which prevented liver injury and reduced the risk of ACLF.

Since $30 \%$ of patients with ACLF have bacterial infections, the intestinal translocation of bacteria or bacterial products would partially explain the development of ACLF without having an active bacterial infection (Takeuchi and Akira, 2010; Wiest et al., 2014; Chen et al., 2015). Intestinal endotoxemia is a major factor causing liver disease progression and multiple organ failure. Endotoxin activates monocytes and macrophages to release a large amount of TNF-a, which binds to the TNF receptor expressed on the hepatocyte membrane, leading to hepatocyte damage. In the present study, XCHD reduces endotoxin and TNF-a. Our previous study has confirmed that baicalein, a main component of XCHD reduces the apoptosis of intestinal mucosal cells, protecting the intestinal barrier injury (Liu et al., 2015).

Accumulating experimental and clinical evidence suggested that prolonged GE and SIT are common in patients with cirrhosis (Theocharidou et al., 2017; Isobe et al., 1994). In addition, prolonged SIT and subsequent small intestinal bacterial overgrowth (SIBO) and bacterial translocation represent a common 
pathogenetic mechanism in the development of diverse complications of cirrhosis (Sánchez et al., 2005; Sato et al., 2012; Scolapio et al., 2002). A previous study demonstrated that the propulsion of intestinal contents by intestinal peristalsis prevented the overgrowth of bacteria (Roland et al., 2015). In the present study, we found that GE and SIT were delayed in ACLF rats. XCHD treatment accelerated the GE and SIT compared to the ACLF group. Similarly, the normal percentage of ISW was significantly improved after than before the XCHD treatment. These findings indicated XCHD improves GE and SIT by improving dysrhythmia of GSW.

Based on the current study design, it was difficult to determine whether XCHD could directly prohibit intestinal bacterial overgrowth and reduce the permeability of the intestinal mucosa. It was also possible that XCHD would have some effects on the intestinal translocation of bacteria or bacterial products via prokinetic effects on gastrointestinal motility. Therefore, additional follow-up studies are needed to prove this hypothesis. Although XCHD is a promising drug for treating ACLF in the future, in-depth studies are essential.

\section{Conclusions}

XCHD prevents the progression of acute-on-chronic hepatic failure partially via the prokinetic effects on gastrointestinal motility to reduce the levels of TNF-a and endotoxin and hepatocyte apoptosis. Although in-depth studies are needed, the current results suggested that preventive therapy with XCHD satisfactorily improves cirrhosis and ACLF survival in rats.

\section{Abbreviations}

ACLF: Acute-on-chronic liver failure

XCHD: Xiao Chai Hu Decoction

ALT: Alanine Aminotransferase

AST: Glutamic oxaloacetylase

TBIL: Total Bilirubin

TNF-a: Tumor necrosis factor alpha

LPS: Lipopolysaccharides

\section{Declarations}

\section{Ethics approval and consent to participate}


The experiment was according to the Guide for the Care and Use of Laboratory Animals (Institute of Laboratory Animal Resources, 1996, Nat. Acad. Press) and approved by the Xi'an Jiaotong University (Xi'an, China) Institutional Animal Care and Use Committee (IACUC).

\section{Consent for publication}

Written informed consent for publication was obtained from all participants

\section{Availability of data and materials}

The datasets used or analysed during the current study are available from the corresponding author on reasonable request.

\section{Competing interests}

The authors declare that they have no competing interests.

\section{Funding}

National major infectious diseases program(2017ZX10203201002-004), National major infectious diseases program(2018ZX10302206-004-010) and Clinical research project of Xi'an Jiaotong University (XJTU1AF-CRF-2015-015) : These projects supported the design of the study and collection, analysis, and interpretation of data.: the design of the study and collection, analysis, and interpretation of data and in writing the manuscript.

\section{Authors' contributions}

FY and JZ: carried out the performance the study, interpretation of data and writing of manuscript. YLW, $S M L, X Z, X J L$ and JZL: carried out some part of the surgery and immunohistochemistry. YL: participated in the design of the study and revised it critically. All authors read and approved the final manuscript.

\section{Acknowledgements}

Not applicable

\section{Authors' information (optional)}

Feng Ye

The 1st Affiliated Hospital of Xi'an Jiaotong University, Shannxi, China; Xi'an 710061, China

No.277, Yanta West Road, Shannxi, China; Xi'an 710061, China

Email: yefeng.jiaotong@163.com 


\section{References}

1. Finkenstedt A, Nachbaur K, Zoller H, Joannidis M, Pratschke J, Graziadei I.W, et al. Acute-on-chronic liver failure: excellent outcomes after liver transplantation but high mortality on the wait list. Liver Transpl. 2013;19: 879-86, .

2. Solé C, Solà E. Update on acute-on-chronic liver failure. Gastroenterol Hepatol. 2018;41:43-53 .

3. Bañares R, Nevens F, Larsen FS, Jalan R, Albillos A, Dollinger M, Saliba F, Sauerbruch T, Klammt S, et al. Extracorporeal albumin dialysis with the molecular adsorbent recirculating system in acute-onchronic liver failure: the RELIEF trial. Hepatology. 2013; 57: 1153-62.

4. Kribben A, Gerken G, Haag S, Herget-Rosenthal S, Treichel U, Betz C, Sarrazin C, Hoste E, Van Vlierberghe $\mathrm{H}$, et al. Effects of fractionated plasma separation and adsorption on survival in patients with acute-on-chronic liver failure. Gastroenterology. 2012; 142: 782-89 .

5. Yuan SF, Jiang T, Sun LH, Zheng RJ, Cao GQ, Ahat NZ, Zhang YX. Use of bone mesenchymal stem cells to treat rats with acute liver failure. Genet Mol Res.2014;30: 6962-80.

6. Huebert RC, Rakela J. Cellular therapy for liver disease. Mayo Clin Proc. 2014; 89: 414-24, .

7. Khanam A, Trehanpati N, Garg V, Kumar C, Garg H, Sharma BC, Sarin SK. Altered frequencies of dendritic cells and IFN-c-secreting T cells with granulocyte colony-stimulating factor (G-CSF) therapy in acute-on- chronic liver failure. Liver Int. 2014; 34: 505-13, .

8. Qi FH, Wang ZX, Cai PP, Zhao L, Gao JJ, Kokudo N, Li AY, Han JQ, Tang W. Traditional Chinese medicine and related active compounds: a review of their role on hepatitis $B$ virus infection. Drug Discov Ther. 2013; 7:212-24, .

9. Xiong XJ, Chu FY, Li HX, He QY. Clinical application of the TCM classic formulae for treating chronic bronchitis. J Tradit Chin Med.2011; 31:69-72, .

10. Dou J, Chen Q, Wang J. Inhibition effect of Chinese herbal medicine on transcription of hepatitis C virus structural gene in vitro.World J Gastroenterol. 2005;11:3619-22, .

11. Li J, Hu R, Xu S, Li Y, Qin Y, Wu Q, Xiao Z. Xiaochaihutang attenuates liver fibrosis by activation of Nrf2 pathway in rats. Biomed Pharmacother. 2017; 96:847-53.

12. Zheng CS, Wu YS, Bao HJ, Xu XJ, Chen XQ, Ye HZ, Wu GW, Xu HF, Li XH, et al. Understanding the polypharmacological anticancer effects of Xiao Chai Hu tang via a computational pharmacological model. Exp Ther Med. 2014; 7:1777-83, .

13. Liu XQ, Hu XJ, Xu HX, Zeng XY. Xiaochaihu Decoction attenuates the vicious circle between the oxidative stress and the ALP inactivation through LPS-catecholamines interactions in gut, liver and brain during CCI4+ethanol-induced mouse HCC. BMC Complement Altern Med. 2013; 13, 375.

14. Cheng PY, Lee YM, Wu YS, Chang TW, Jin JS, Yen MH. Protective effect of baicalein against endotoxic shock in rats in vivo and in vitro. Biochem Pharmacol. 2007; 73:793-804.

15. Miyasaki Y, Rabenstein JD, Rhea J, Crouch ML, Mocek UM, Kittell PE, Morgan MA, Nichols WS, Van Benschoten $\mathrm{MM}$, et al. Isolation and characterization of antimicrobial compounds in plant extracts against multidrug-resistant acinetobacter baumannii. PLoS One. 2013; 22, e61594. 
16. Qiao H, Tong Y, Han H, Xu W, Ren Z, Ouyang J, Chen Y. A novel therapeutic regimen for hepatic fibrosis using the combination of mesenchymal stem cells and baicalin. Die Pharmazie.2011; 66:37-43.

17. Pan TL, Wang PW, Leu YL, Wu TH, Wu TS. Inhibitory effects of scutellaria baicalensis extract on hepatic stellate cells through inducing G2/M cell cycle arrest and activating ERK-dependent apoptosis via Bax and caspase pathway. J Ethnopharmacol. 2012;139:829-37.

18. Shen YC, Chiou WF, Chou YC, Chen CF. Mechanisms in mediating the anti-inflammatory effects of baicalin and baicalein in human leukocytes. Eur J Pharmacol. 2003; 465:171-81.

19. Kubo M, Matsuda H, Tanaka M, Kimura Y, Okuda H, Higashino M, Tani T, Namba K, Arichi S. Studies on scutellariae radix. VII. Anti-arthritic and anti-inflammatory actions of methanolic extract and flavonoid components from scutellariae radix. Chem Pharm Bull.1984; 32:2724-729.

20. Yang LP, Sun HL, Wu LM, Guo XJ, Dou HL, Tso MO, Zhao L, Li SM. Baicalein reduces inflammatory process in a rodent model of diabetic retinopathy. Invest Ophthalmol Vis Sci. 2009; 50:2319-327.

21. Yun MY, Yang JH, Kim DK, Cheong KJ, Song HH, Kim DH, Cheong KJ, Kim YI, Shin SC. Therapeutic effects of baicalein on atopic dermatitis-like skin lesions of NC/Nga mice induced by dermatophagoides pteronyssinus. Int Immunopharmacol.2010; 10:1142-48.

22. Dou W, Mukherjee S, Li H, Venkatesh M, Wang H, Kortagere S, Peleg A, Chilimuri SS, Wang ZT, Feng $\mathrm{Y}$, et al. Alleviation of gut inflammation by $\mathrm{Cdx2/Pxr}$ pathway in a mouse model of chemical colitis. PLoS One. 2012; 7, e36075.

23. Su GY, Yang JY, Wang F, Ma J, Zhang K, Dong YX, Song SJ, Lu XM, Wu CF. Antidepressant-like effects of Xiaochaihutang in a rat model of chronic unpredictable mild stress. J Ethnopharmacol. 2014; 152:217-26.

24. Amacher DE. Strategies for the early detection of drug-induced hepatic steatosis in preclinical drug safety evaluation studies. Toxicology. 2011; 79:10-8.

25. Hernández-Muñoz R, Díaz-Muñoz M, López V, López-Barrera F, Yáñez L, Vidrio S, Aranda-Fraustro A, Chagoya de Sánchez V. Balance between oxidative damage and proliferative potential in an experimental rat model of $\mathrm{CCl} 4$-induced cirrhosis: protective role of adenosine administration. Hepatology. 1997; 26:1100-10.

26. L.W. Weber, M. Boll, A. Stampfl. Hepatotoxicity and mechanism of action of haloalkanes: carbon tetrachloride as a toxicological model. Crit Rev Toxicol. 2003; 33: 105-36.

27. Furuya S, Chappell GA, Iwata Y, Uehara T, Kato Y, Kono H, Bataller R, Rusyn I. A mouse model of alcoholic liver fibrosis-associated acute kidney injury identifies key molecular pathways. Toxicol Appl Pharmacol.2016; 310:129-39.

28. Liu XH, Chen Y, Wang TL, Lu J, Zhang LJ, Song CZ, Zhang J, Duan ZP. Establishment of a D-Gal /LPS induced acute-on-chronic liver failure model in rats. Zhonghua Gan Zang Bing Za Zhi. 2007; 15:771-75.

29. Arroyo V, Moreau R, Jalan R, Ginès P; EASL-CLIF Consortium CANONIC Study. Acute-on-chronic liver failure: A new syndrome that will re-classify cirrhosis. J Hepatol. 2015; 62: 131-43. 
30. Ruiz-Margáin A, Pohlmann A, Ryan P, Schierwagen R, Chi-Cervera LA, Jansen C, Mendez-Guerrero O, Flores-García NC, Lehmann J, et al. Fibroblast growth factor 21 is an early predictor of acute-onchronic liver failure in critically ill patients with cirrhosis. Liver Transpl. 2018; 24:595-605.

31. Arroyo V, Moreau R, Kamath PS, Jalan R, Ginès P, Nevens F, Fernández J, To U, García-Tsao G, Schnabl B. Acute-on-chronic liver failure in cirrhosis. Nat Rev Dis Primers. 2016; 2:16042.

32. Clària J, Arroyo V, Moreau R.The Acute-on-Chronic Liver Failure Syndrome, or When the Innate Immune System Goes Astray. J Immunol.2016; 197: 3755-61.

33. Baggiolini M. Chemokines in pathology and medicine. J Intern Med. 2001; 250:91-104.

34. Li H. Advances in anti hepatic fibrotic therapy with Traditional Chinese Medicine herbal formula. $\mathrm{J}$ Ethnopharmacol. 2020; 251:112442.

35. Gressner, O.A., Weiskirchen, R., Gressner, A.M. Biomarkers of liver fibrosis: clinical translation of molecular pathogenesis or based on liver-dependent malfunction tests. Clin Chim Acta. 2007; 381:107-13.

36. Wu T, Li J, Shao L, Xin J, Jiang L, Zhou Q, Shi D, Jiang J, Sun S, Jin L, Ye P, Yang L, Lu Y, Li T, Huang J, Xu X, Chen J, Hao S, Chen Y, Xin S, Gao Z, Duan Z, Han T, Wang Y, Gan J, Feng T, Pan C, Chen Y, Li H, Huang Y, Xie Q, Lin S, Li L, Li J; Chinese Group on the Study of Severe Hepatitis B (COSSH). Development of diagnostic criteria and a prognostic score for hepatitis B virus-related acute-onchronic liver failure. Gut. 2018;67:2181-91.

37. Horvatits T, Drolz A, Roedl K, Rutter K, Ferlitsch A, Fauler G, Trauner M, Fuhrmann V. Serum bile acids as marker for acute decompensation and acute-on-chronic liver failure in patients with noncholestatic cirrhosis. Liver Int. 2017; 37:224-31.

38. Takeuchi O, Akira S. Pattern recognition receptors and inflammation. Cell. 2010; 140: 805-20.

39. Wiest R, Lawson M, Geuking M. Pathological bacterial translocation in liver cirrhosis. J Hepatol. 2014; 60: 197-209.

40. Chen P, Stärkel J R, Turner S B, Schnabl B. Dysbiosis-induced intestinal inflammation activates TNFRI and mediates alcoholic liver disease in mice. Hepatology.2015; 61: 883-94.

41. Liu Y, Ye F, Zou WJ, Sun Y, Wang R, Han PP, Zhang Z, Yang XL, Liu X. Baicalein reduces the occurrence of cirrhotic endotoxemia by reducing intestinal mucosal apoptosis. BMC Complement Altern Med. 2015;15:161.

42. Adebayo D, Morabito V, Andreola F, Pieri G, Luong TV, Dhillon A, Mookerjee R, Jalan R. Mechanism of cell death in acute-on-chronic liver failure: a clinico-pathologic-biomarker study. Liver Int.2015; 35:2564-74.

43. Cai J, Han T, Nie C, Jia X, Liu Y, Zhu Z, Gao Y. Biomarkers of oxidation stress, inflammation, necrosis and apoptosis are associated with hepatitis B-related acute-on-chronic liver failure. Clin Res Hepatol Gastroenterol. 2016;40:41-50.

44. Bechmann LP, Marquitan G, Jochum C, Saner F, Gerken G, Canbay A. Apoptosis versus necrosis rate as a predictor in acute liver failure following acetaminophen intoxication compared with acute-onchronic liver failure. Liver International, 2008; 28: 713-16. 
45. Rastogi A, Kumar A, Sakhuja P, Bihari C, Gondal R, Hissar S, Garg H, Sarin SK. Liver histology as predictor of outcome in patients with acute-on-chronic liver failure (ACLF). Virchows Arch. 2011; 459:121-27.

46. Saich R, Selden C, Rees M, Hodgson H. Characterization of pro-apoptotic effect of liver failure plasma on primary human hepatocytes and its modulation by molecular adsorbent recirculation system therapy. Artif Organs. 2007; 31:732-42.

\section{Figures}

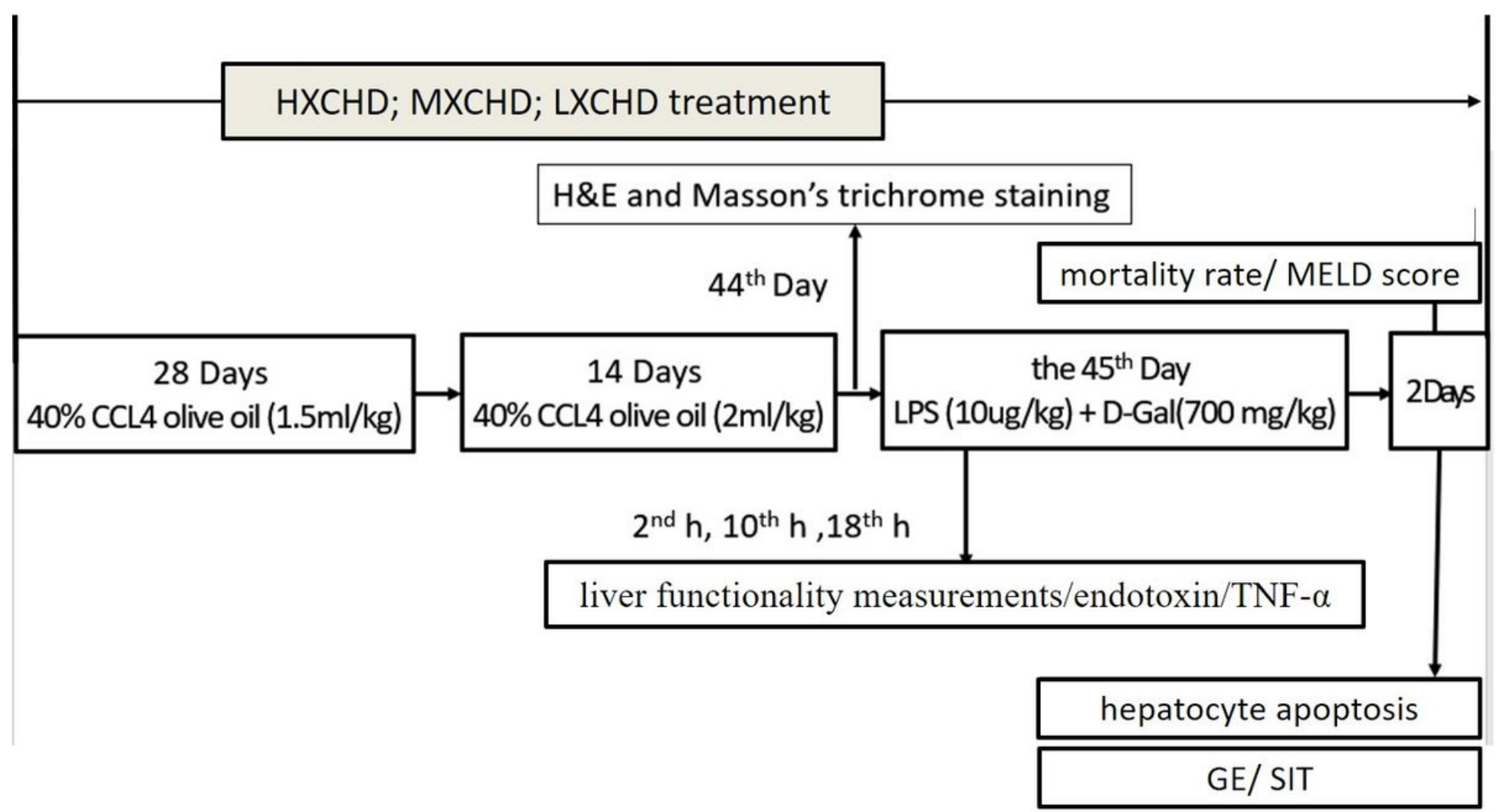

\section{Figure 1}

Experimental protocols. HXCHD: high dose of Xiao-chai-hu Decoction; MXCHD: middle dose of Xiao-chaihu Decoction; LXCHD: Iow dose of Xiao-chai-hu Decoction.
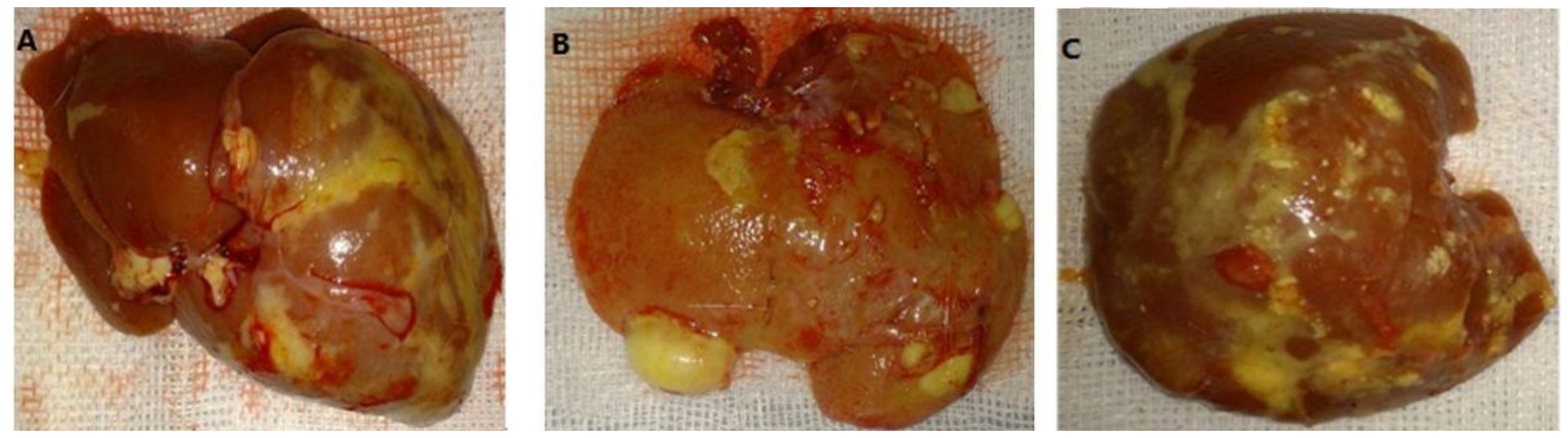
Figure 2

Liver of rats. A. normal; B. cirrhosis model; C. ACLF model. ACLF: Acute-on-chronic Liver Failure.
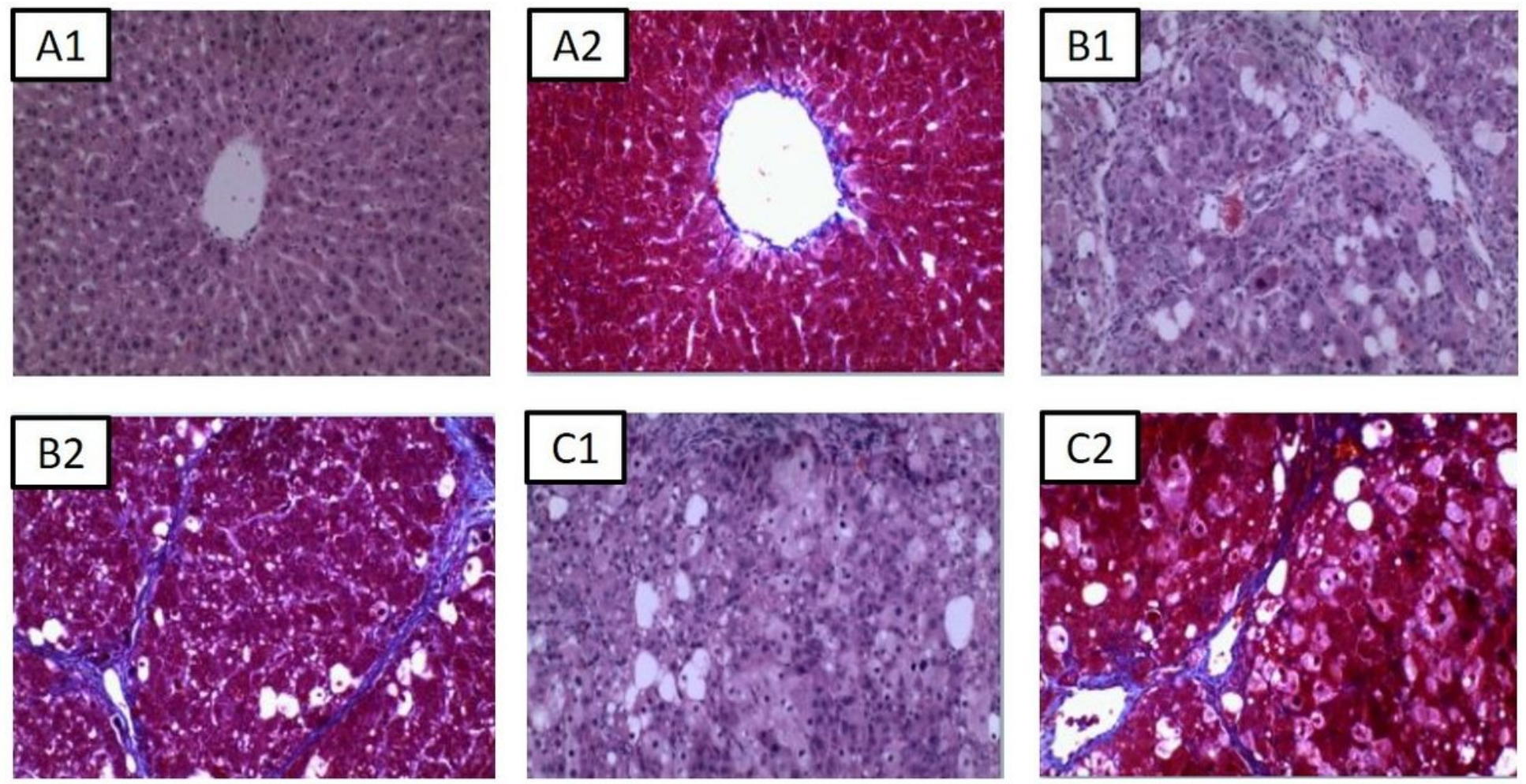

\section{Figure 3}

The pathological changes of liver tissue in each group (×10). 1: H\&E staining; 2 : Masson's trichrome staining. A1 and A2: The pathological changes of liver tissue in normal group; B1 and B2: The pathological changes of liver tissue in cirrhosis model group; $\mathrm{C} 1$ and $\mathrm{C} 2$ : The pathological of liver tissue in ACLF model. ACLF: Acute-on-chronic Liver Failure. 

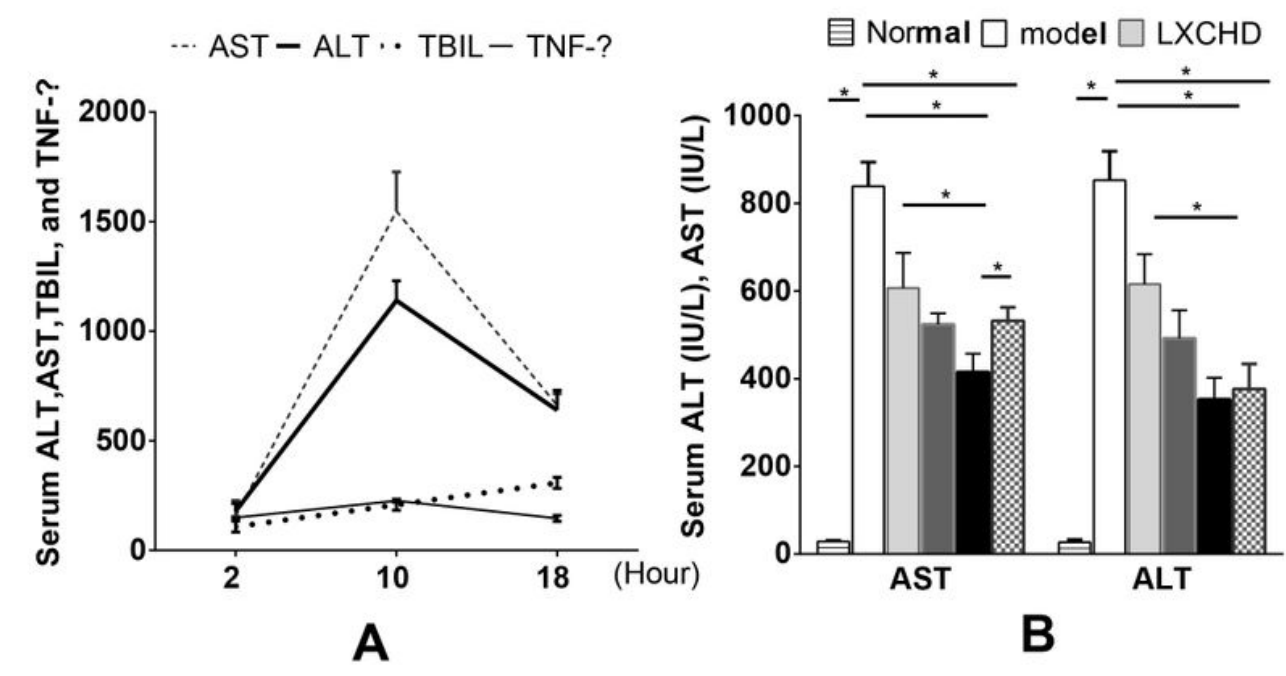

$\square \mathrm{MXCHD} \square \mathrm{HXCHD} \otimes \mathrm{PP}$
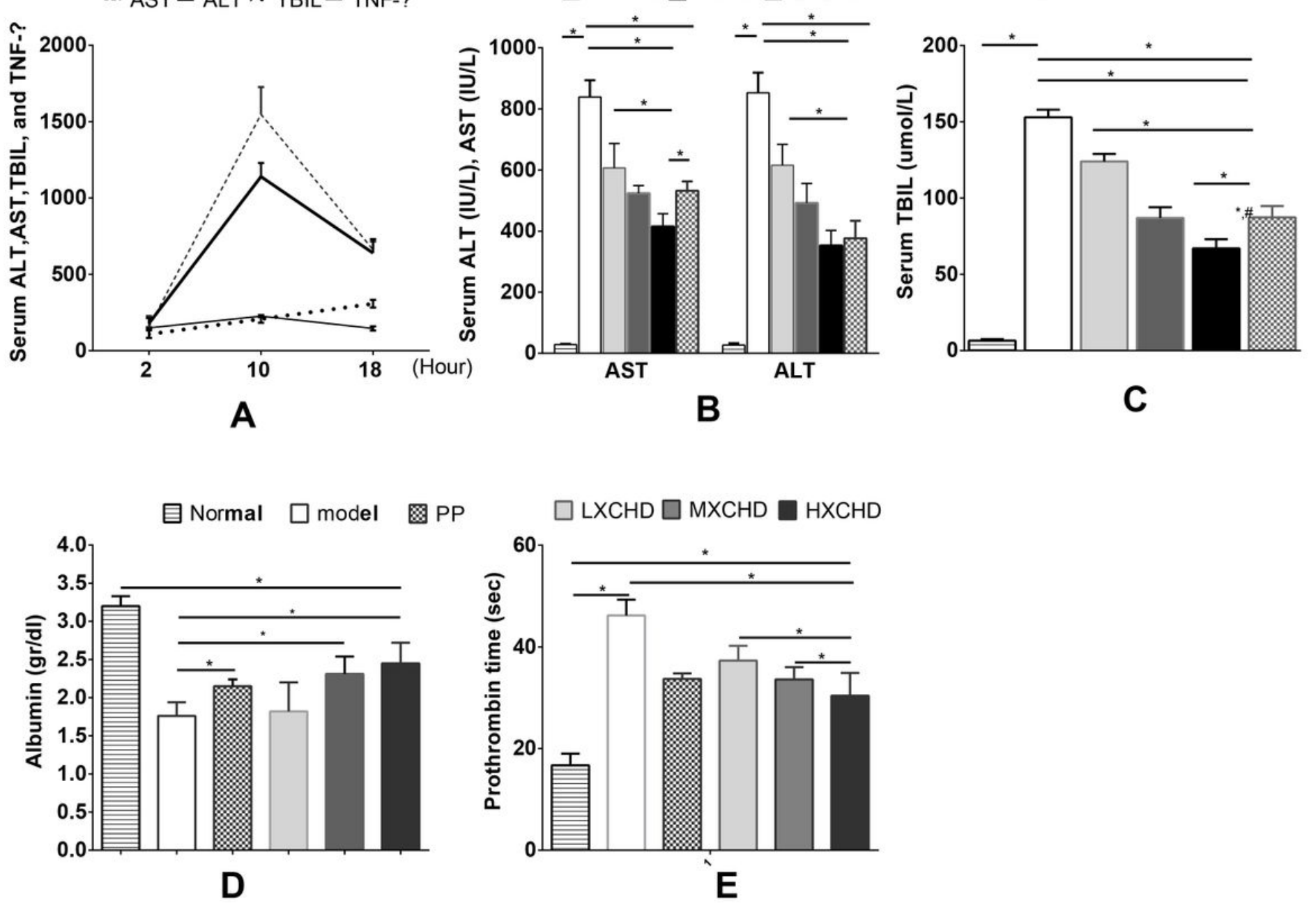

Figure 4

Comparison of the serum levels of liver functionality measurements in each group. A: serum level of ALT, AST, TBIL, and TNF-a in model rats after acute attack with LPS/ D-Gal; B. serum level of ALT and AST; C. serum level of TBIL; D. serum level of albumin; E. serum level of prothrombin time. Asterisk: significant at $p<0.05$.
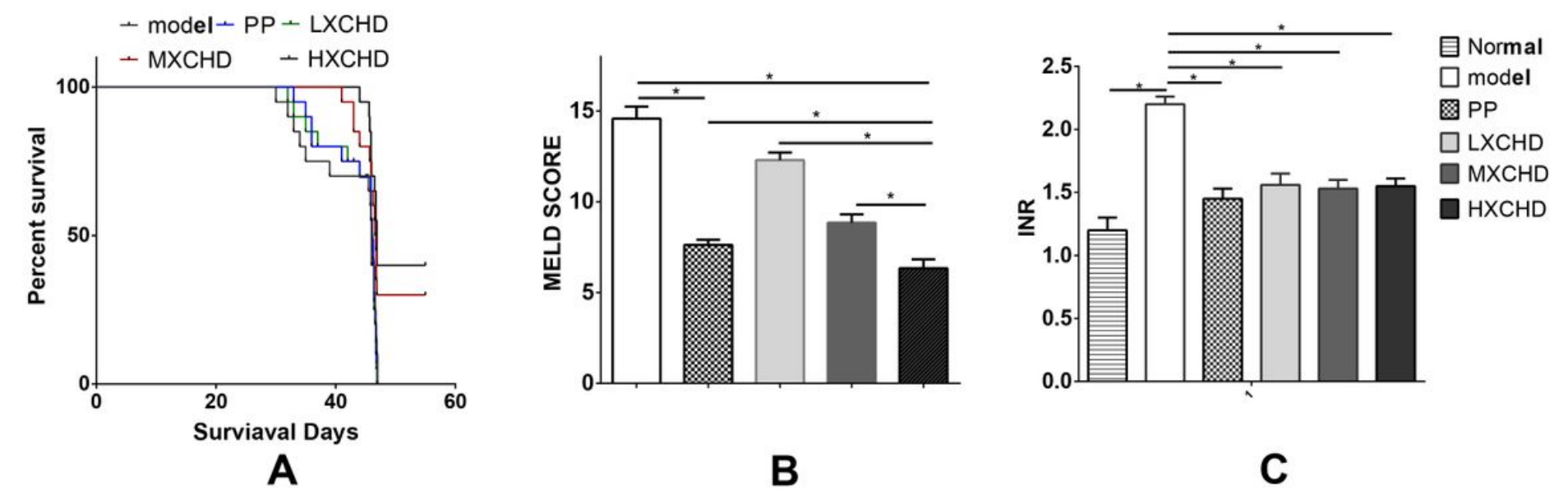

B

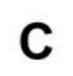


Figure 5

Comparison of the survival rate, rat-adapted MELD score and INR in each group. A: Kaplan-Meier representation of survival of ACLF rats. B: Comparison of MELD score; C: International Normalized Ratio. Asterisk: significant at $p<0.05$.

A

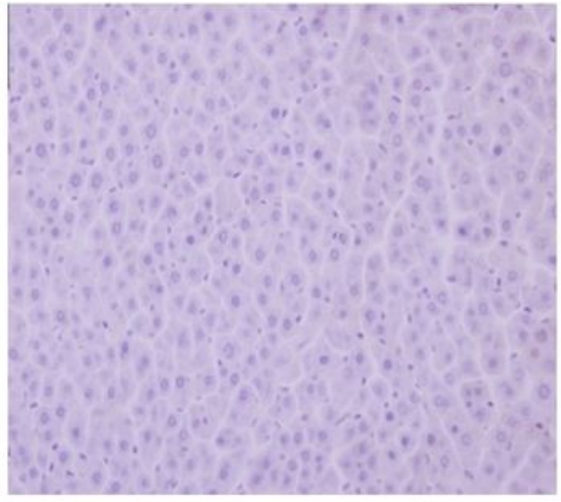

$\mathrm{D}$

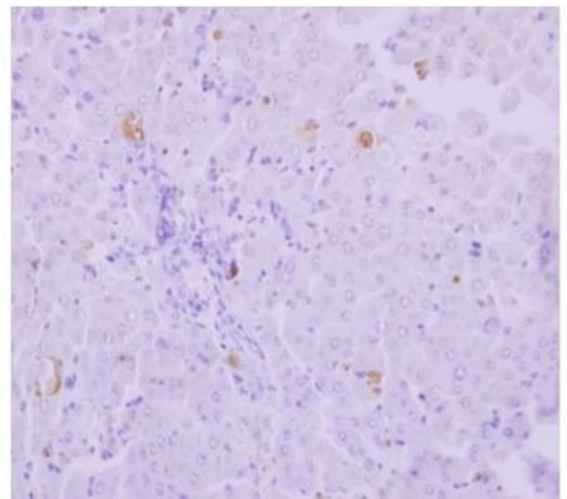

B

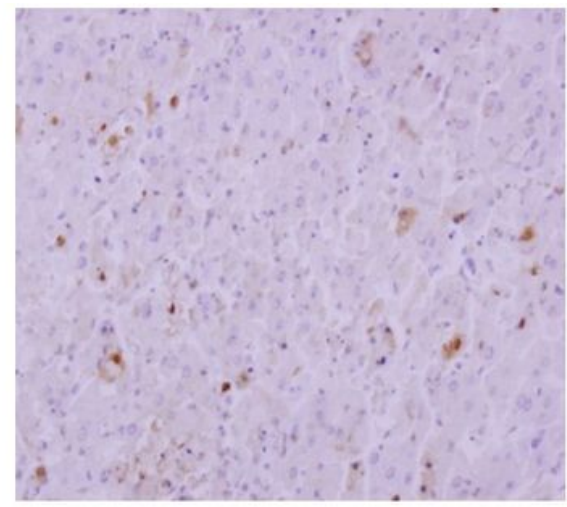

E

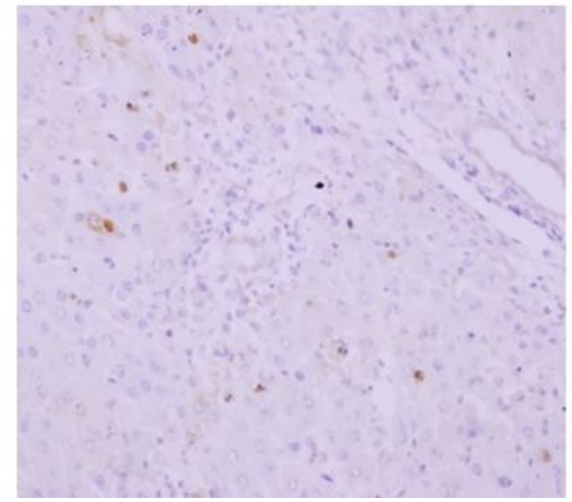

C

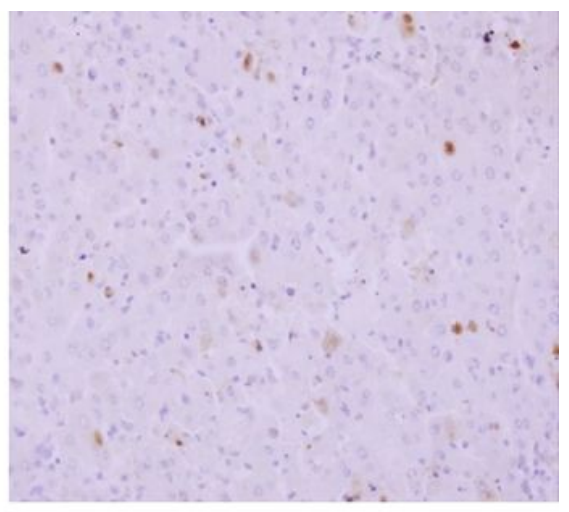

$\mathrm{F}$

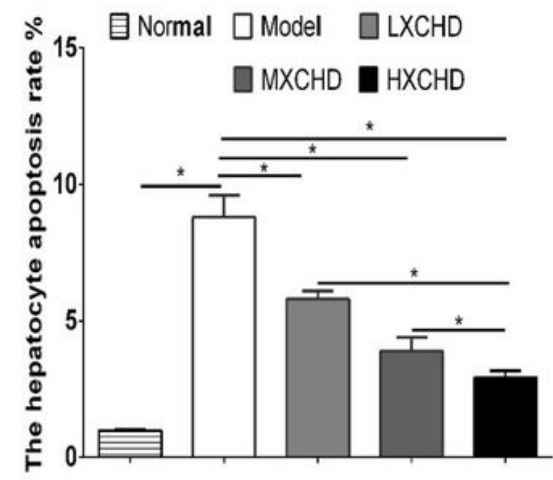

Figure 6

Comparison of the ratios of the area of hepatocyte apoptosis in each group (TUNLE staining, $10 \times 40$ ). A: normal group; B: model group; C: LXCHD treatment group; D: MXCHD treatment group; E: HXCHD treatment group. F: Comparison of the hepatocyte apoptosis rate. Asterisk: significant at $p<0.05$.

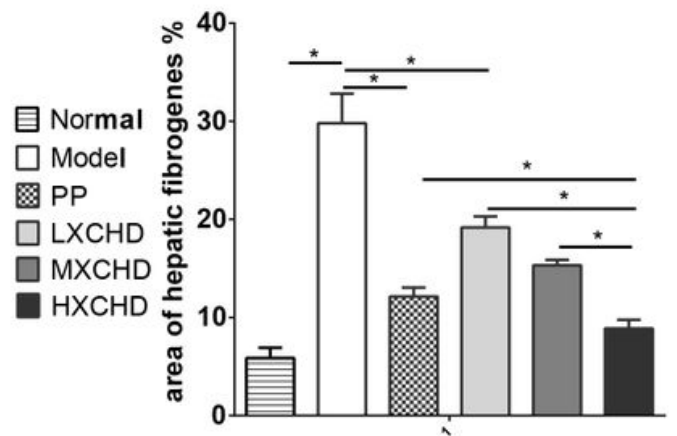

A

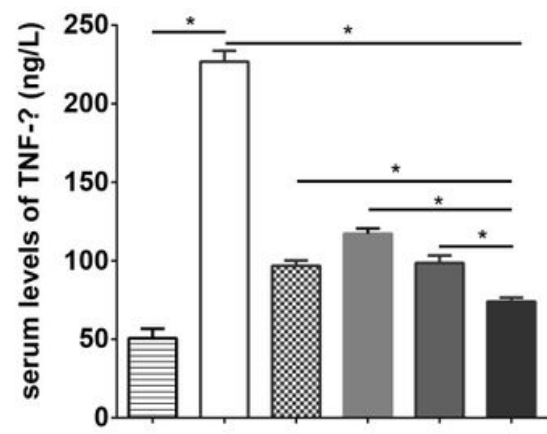

B

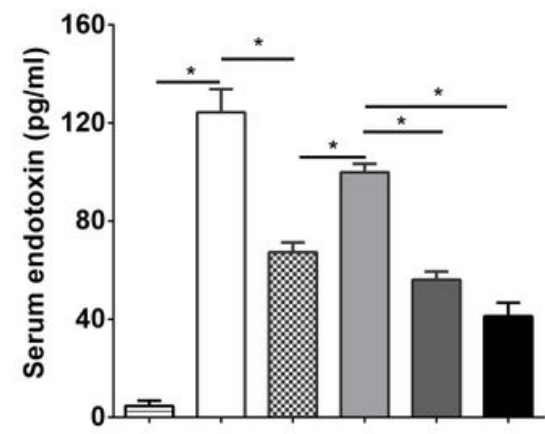

C

Figure 7 
Comparison of the serum levels of TNF-a and endotoxin in each group. A. area of hepatic fibrogenesis; B. serum levels of TNF-a; $C$. serum levels of endotoxin. Asterisk: significant at $p<0.05$.

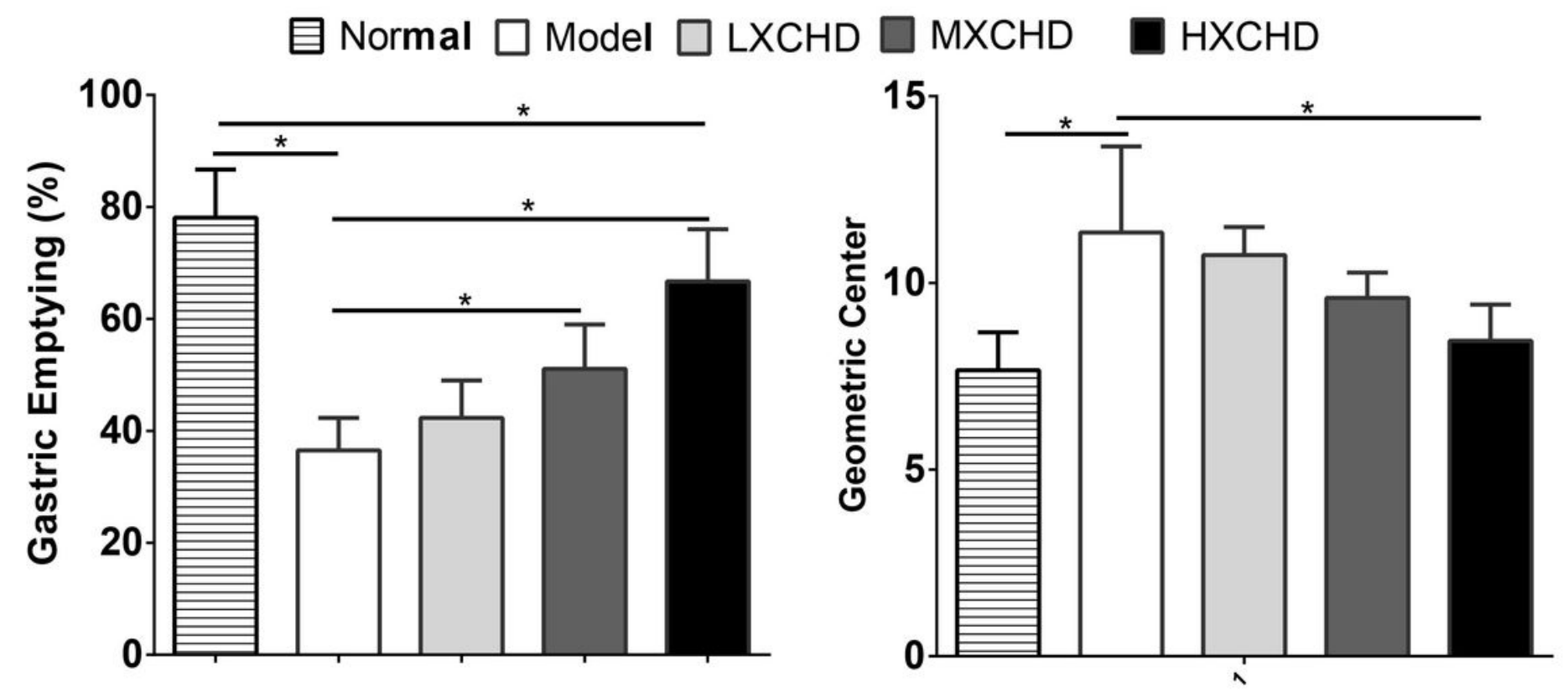

Figure 8

Prokinetic effects of XCHD on motility in each group. A: gastric emptying; B. geometric center. Asterisk: significant at $p<0.05$. 
A

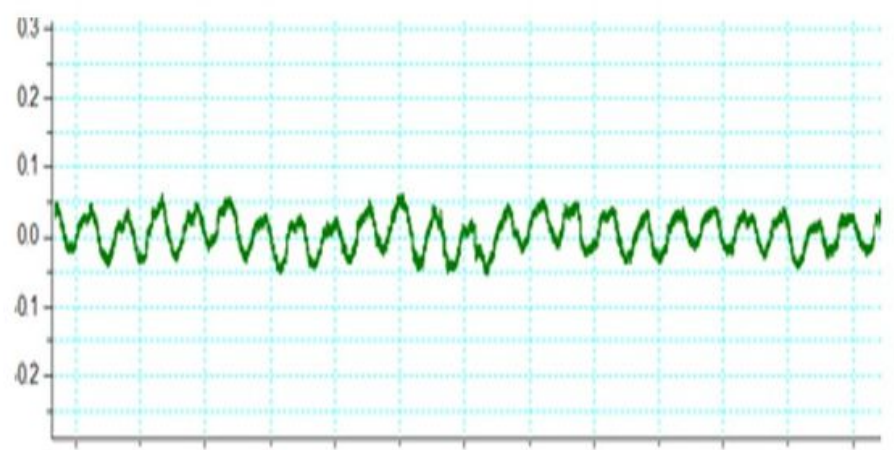

C

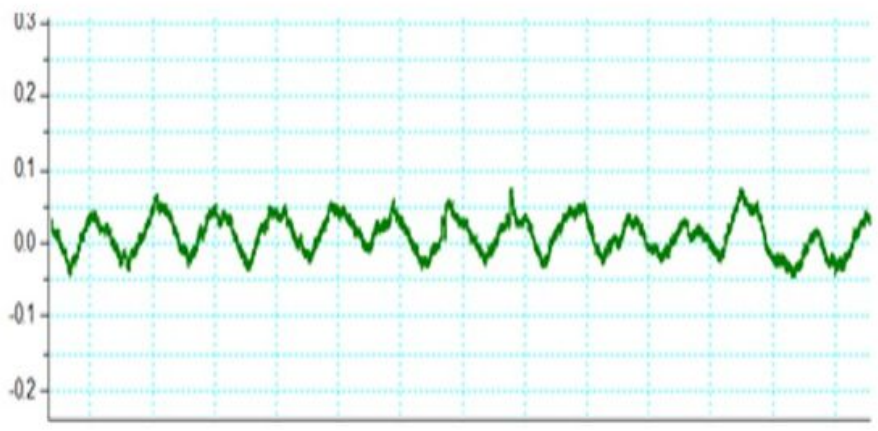

B

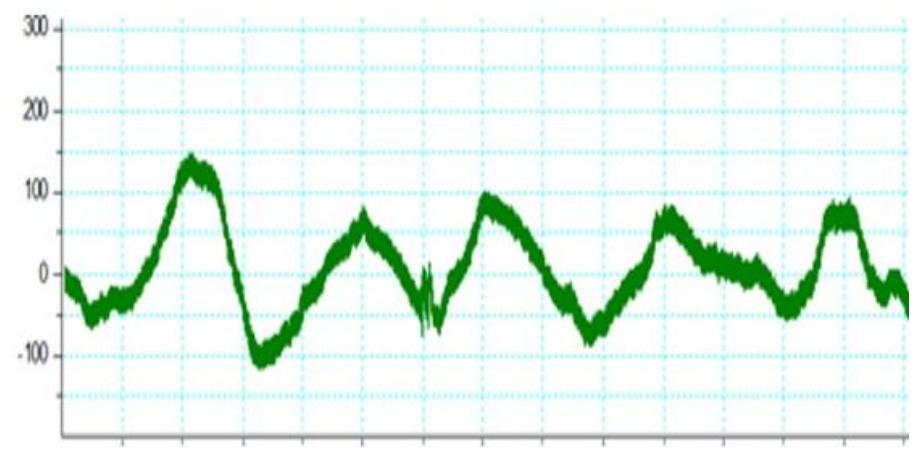

D

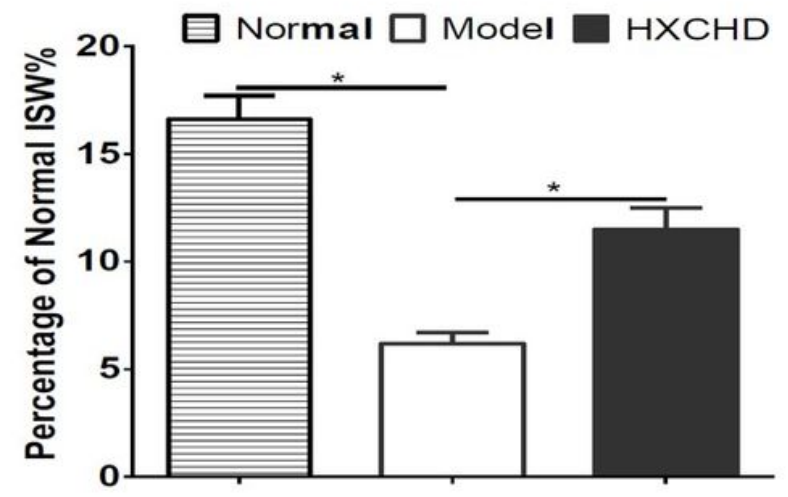

Figure 9

The typical signal traces of slow waves of ileum in each group (20 seconds): A. normal group; B. ACLF group; C. HXCHD group; D. percentage of normal ISW\%. Asterisk: significant at $\mathrm{p}<0.05$. 